\title{
Laparoscopy in elective and emergency management of ovarian pathology in children and adolescents
}

\author{
Andrzej Grabowski, Wojciech Korlacki, Michat Pasierbek \\ Pediatric Surgery Department, Medical University of Silesia, Zabrze, Poland
}

Videosurgery Miniinv 2014; 9 (2): 164-169

DOI: $10.5114 /$ wiitm.2014.41626

\begin{abstract}
Introduction: Ovaries are one of the most common locations of tumor masses in children. Some of them require surgery due to the risk of malignancy or necrosis. This organ seems to be ideal for the laparoscopic approach.

Aim: To evaluate the usefulness of laparoscopy in surgery of lesions located in the ovaries in patients under 18 years of age and assess the risk of changes in the ovaries in girls with acute abdominal symptoms.

Material and methods: Retrospective evaluation of hospital records of the period 1996-2012 from a single hospital was performed. 105 laparoscopic procedures of ovarian pathology in patients aged 0-18 (mean: 13.5) years were reviewed. The overall sample was divided into groups depending on the indication and mode of surgery. Group I: elective or emergency surgery, imaging findings of ovarian cysts bigger than $5 \mathrm{~cm}$ or causing pain. Group II: elective surgery, the ovarian tumor visible in imaging (solid mass or mixed). Group III: treatment for acute abdomen, without visible ovarian pathology in the preoperative imaging studies. Group IV: elective treatment of other indications, incidental finding.

Results: There were no deaths or major complications. There were no conversions. Average length of hospital stay after surgery was 2.5 days. The risk of appendicitis in patients referred for surgery due to ovarian cysts visualized in ultrasound, in the factual absence of ovarian pathology (false positive ultrasound), in the presented material was $5.2 \%$. The risk of lesions in the ovaries in patients operated on due to acute abdominal pain, with no findings in the pre-operative ultrasound (false negative ultrasound), in the presented material was $7.4 \%$. The risk of coexistence of changes in the ovaries with appendicitis found during the procedure due to acute abdominal pain in the study group was $6 \%$.

Conclusions: The laparoscopic treatment for ovarian masses is safe and efficient. The risk of wrong preoperative diagnosis (ovary mass vs. appendicitis) is in any direction between 5 and $8 \%$, which is a number large enough to be taken into consideration when surgical training and legislation is concerned.
\end{abstract}

Key words: ovary, laparoscopy, ovarian mass, ovarian cyst, ovarian torsion.

\section{Introduction}

Ovaries are one of the most common locations of tumor masses in children. Such changes are observed predominantly in neonates and infants and girls in peripubertal age. In both cases, $97 \%$ of the lesions are benign, with a large majority of simple cysts, which definitely distinguishes the pediatric population from adult patients [1]. In both groups an asymptomatic cyst with negative germ cell tumor markers is not an indication for surgical treatment, and in some cases hormonal therapy is indicated [2, 3]. 
Any pathology in the ovary may, however, enter the symptomatic stage. With increasing cyst diameter the risk of ovarian torsion increases - the size limit was established at $5 \mathrm{~cm}$. We must take into consideration ovarian pathology as a cause of chronic or acute pain, including cases requiring emergency intervention. Disclosure of the tumor during surgery for other indications is also possible. Also, due to the ambiguous findings obtained by preoperative imaging, it may be necessary to implement the treatment in accordance with oncological guidelines and tumor resection [1].

\section{Aim}

The study was performed to evaluate the usefulness of laparoscopy in surgery of lesions located in the ovaries in patients under 18 years of age. We also wanted to assess the risk of changes in the ovaries in girls with acute abdominal symptoms and the risk of appendicitis in those with supposed or factual ovarian masses.

\section{Material and methods}

The work was based on a retrospective analysis of hospital records from a single tertiary center. In the period 1996-2012, 105 laparoscopic procedures of ovarian pathology in patients aged 0-18 years (mean: 13.5 years) were performed. This population was divided into four age groups: children under one year of age, children under 9 years of age, girls between 10 and 15 years of age, and those between 16 and 18 years of age. Table I shows the distribution of age groups. At the time of the survey emergency operational treatment for acute abdominal pain was carried out in 907 patients, including 415 girls.

The overall sample was also divided into groups depending on the indication and mode of surgery (Table II): group I: elective or emergency surgery, imaging findings of ovarian cysts bigger than $5 \mathrm{~cm}$

Table I. Distribution of age groups

\begin{tabular}{|lcc|}
\hline Age group [years] & Number & Percentage \\
\hline $0-1$ & 6 & 5.7 \\
\hline $2-9$ & 8 & 7.6 \\
\hline $10-15$ & 48 & 45.7 \\
\hline $16-18$ & 43 & 40.9 \\
\hline
\end{tabular}

or causing pain; group II: elective surgery, the ovarian tumor visible in imaging (solid mass or mixed); group III: treatment for acute abdomen, without the visibility of ovarian pathology in the preoperative imaging studies; group IV: elective treatment of other indications, incidental finding.

We evaluated the type of ovarian pathology based on the result of the histopathological examination, length of hospital stay, and number and type of complications in the perioperative period.

We assessed: the risk of the presence of malignancy in the treated population, the incidence of ovarian pathology in patients targeted for surgery because of the preoperative ultrasound imaging findings in relation to the total number of operations performed in girls due to symptoms of acute abdomen, the frequency of appendicitis in patients with preoperative image of ovarian pathology.

\section{Results}

Postoperative diagnosis, confirmed by histopathological examination in the case of resection, is indicated in Tables II-VI.

Group I (Table III). In all cases of simple cysts (including ruptured ones) the wall of the cyst was excised and the padding removed, saving the rest of the ovary. The histopathological examination confirmed the benign picture of the lesions. In 3 cases of ovarian torsion with mild ischemic signs the proper blood supply returned to the organ after derotation. In these cases no specimens were collected, so the diagnosis has no histological verification. However, there was no evidence for features of tumorous organ reconstruction. In 2 cases, the severity of necrotic lesions made excision of the ovary necessary. Histological verification confirmed ischemia and necrosis. In 1 patient the ovary was torn off and floated freely in the peritoneal cavity. It was a patient in the second month of life, observed from birth due to a prenatal finding of right ovarian tumor (cyst).

Table II. Number of patients in test groups

\begin{tabular}{|lcc|}
\hline Group & Number & Percentage \\
\hline I & 57 & 54.2 \\
\hline II & 13 & 12.3 \\
\hline III & 33 & 31.4 \\
\hline IV & 2 & 1.9 \\
\hline
\end{tabular}


Table III. Postoperative diagnosis in group I

\begin{tabular}{|lc|}
\hline Type of pathology & Number \\
\hline Simple cyst rupture & 5 \\
\hline Ovarian torsion (with or without the cyst) & 6 \\
\hline Simple cyst & 34 \\
\hline Polycystic ovary syndrome (PCOS) & 6 \\
\hline Ovarian tumor (mature teratoma) & 3 \\
\hline Appendicitis & 3 \\
\hline
\end{tabular}

Table V. Postoperative diagnosis in group III

\begin{tabular}{|lc|}
\hline Type of pathology & Number \\
\hline Simple cyst rupture & 4 \\
\hline Ovarian torsion & 3 \\
\hline Simple cyst & 21 \\
\hline Polycystic ovary syndrome & 5 \\
\hline Appendicitis & 2 \\
\hline
\end{tabular}

The $\alpha$-fetoprotein (AFP) was initially elevated but then gradually lowered (to within the physiological limits), which made us wait. In a subsequent ultrasound scan displacement of the tumor into the left body side was revealed. She was promptly scheduled for surgery, and the tumor was laparoscopically removed. Histopathological examination revealed the presence of a simple cyst in the ovary.

Organ surface scarification was performed in all cases of polycystic ovary syndrome.

Tumors were removed in accordance with the oncological protocol, in a nylon bag and through the extended incision for the lowest trocar. The washings from the peritoneal cavity were collected and a specimen from the contralateral ovary was taken.

Inflamed appendixes, where infiltration over the cecum mimicked a tumor, were typically removed. In none of the 3 cases were there macroscopic changes in the ovaries.

Group II (Table IV). In 11 cases of neoplastic lesions patients underwent resection according to the oncological protocol. Resection was also performed in other cases. In both cases of malignant lesions and 2 cases of benign tumors the increase of the activity of germinal tumor markers in peripheral blood was observed.

The case of a patient with a congenital defect is especially worth mentioning. In all imaging studies
Table IV. Postoperative diagnosis in group II

\begin{tabular}{|lc|}
\hline Type of pathology & Number \\
\hline Mature teratoma & 9 \\
\hline $\begin{array}{l}\text { Teratoma with malignant component (yolk sac } \\
\text { tumor) }\end{array}$ & 2 \\
\hline $\begin{array}{l}\text { Fallopian tube birth defect with secondary } \\
\text { ovarian edema }\end{array}$ & 1 \\
\hline Simple cyst & 1 \\
\hline
\end{tabular}

Table VI. Postoperative diagnosis in group IV

\begin{tabular}{|lc|}
\hline Type of pathology & Number \\
\hline Simple cyst & 2 \\
\hline
\end{tabular}

performed preoperatively (ultrasound, computed tomography - CT and magnetic resonance imaging - MRI) a tumor of the ovary was observed. Aligned with it but not in a direct relationship an ovoid mass was found and described as a stationary extended sigmoid loop. The patient presented pain, which was associated with the described loop of sigmoid colon (dolichosigma). The key factors for the decision of resection were the elevated levels of the marker CA-125 $(151 \mu / \mathrm{l})$ and the negative results of the AFP and $\beta$-HCG exam. We confirmed tumorous ovary during the operation. The supposed sigmoid loop was in close association with a wide and distended fallopian tube which was obviously not a part of the intestines. The enlarged ovary with fallopian tube and adherent mass was excised and evacuated in a nylon bag through the extended cut for the lowest trocar. Paraffin study revealed the nature of the change: a fragment described as a swollen fallopian tube was the left corner of a double uterus with endometrium in the proliferative phase. Neoplastic foci were not found. Within the ovary only edematous tissues were found. Neoplastic foci were not found. Swelling and hyperemia were probably caused by the compression due to hypertrophy of the uterus filled with the shed but not evacuated endometrium. The hyperemia in the organ may have caused the increased level of CA-125.

Group III (Table V). In this group, the procedure was similar to that in group I. In cases of ovarian torsion in 2 cases it was necessary to remove the 
organ; one ovary was saved by derotation. In this group appendicitis coincided with changes in the ovaries $(1 \times$ ruptured cyst, $1 \times$ ovarian torsion). But what is worth noting is that none of those cases was revealed in the preoperative ultrasound scan and all patients presented acute abdominal pain.

Group IV (Table VI). The fourth group consists of the two patients operated on electively due to gastroesophageal reflux with esophagitis. The cyst was found intraoperatively, and because of its size, in both cases exceeding $5 \mathrm{~cm}$, it was decided to perform the ovary sparing cyst resection.

In the described material there were no deaths. There was no conversion to laparotomy at the excisional stage. The incisions made to evacuate removed organs (when a solid mass was resected) did not exceed $5 \mathrm{~cm}$ and according to the literature are not conversions [4]. Their location was chosen considering the best cosmetic effect. There were no serious intraoperative bleeding events requiring transfusion or other extra treatment. There were no adverse events associated with surgical technique in the intraoperative and postoperative hospitalization period. Average length of hospital stay after surgery was 2.5 days.

Throughout the material the ratio of benign to malignant character of ovarian lesions was 103 : 2 (proportion of malignant lesions: 1.9\%).

The risk of appendicitis in patients referred for surgery due to ovarian cysts visualized in ultrasound, in the factual absence of ovarian pathology (false positive ultrasound), in the presented material was $5.2 \%$ (3 of 57 ).

The risk of lesions in the ovaries in patients operated on due to acute abdominal pain, with no findings in the pre-operative ultrasound (false negative ultrasound), in the presented material was 7.4\% (31 of 415).

The risk of coexistence of changes in the ovaries with appendicitis found during the procedure due to acute abdominal pain in the study group was $6 \%$ (2 of 33).

Among the 105 patients with pathology of ovaries, ovarian torsion occurred in 9 (8.5\%). It also represents $2.1 \%$ of the total population of girls operated on due to abdominal pain. Sensitivity of ultrasound in the assessment of ovarian pathology in this case can be estimated at $66 \%$, but the specificity at zero (no suggested ovarian torsion even in cases of visualized ovary enlargement). In all patients, the pre- dominant symptom was pain localized in the lower abdomen.

\section{Discussion}

Considering the results it can be concluded that laparoscopy is a safe method of ovarian surgery in children. The ovary, because of its mobility, is an organ that is ideally suited for laparoscopic surgery. In turn, the deep position in the pelvis hinders treatment, especially when access is primarily targeted at the appendix. Laparoscopy allows one to diagnose the changes in the ovaries and perform surgery without changing the access [5-8].

The laparoscopic treatment of simple cysts is not limited by their diameter. Although there were not described any significant complications even after spillage of the contents of the cyst into the abdominal cavity, laparoscopy enables decompression of the content prior to excision under direct vision by percutaneous puncture or insertion of a suction catheter into the lumen of the cyst $[9,10]$.

The percentage of observed malignant pathology is consistent with data from the literature, which, with significant disparities in favor of benign findings, allows safe laparoscopic entry of lesions without evidence of malignancy in the preoperative studies $[5,6]$. It is, however, compulsory to maintain the oncological protocol in the case of each mass which is at least partially solid [11, 12]. Blood marker tests performed before the procedure showed high specificity for germinal tumors, but led to erroneous conclusions in the case of congenital defects, which, along with confusing results of imaging studies, resulted in an unnecessary broadening of the resection. One should keep this in mind when considering each ovary excision [13].

We believe that the dimension of the mass is a restriction for laparoscopy only to a certain extent. Although the need to evacuate the tumor intact makes it necessary to make an additional incision when the diameter exceeds the trocar wound, deteriorating the cosmesis, the better visualization of the pelvic cavity in laparoscopy makes surgery more efficient and radical. This does not apply to those lesions which involve the entire pelvic cavity and/or lose their mobility.

On the other hand, recently published data show the efficiency of less minimal than conventional three-trocar surgery for resections in elective and 
emergency surgery [14-16]. Though the cited materials deal with other organs, retrospectively we have a strong conviction that the proposed access could be suitable for ovariectomy or ovary-sparing surgery. The one-, two- or three-port protocol seems be ideal for diagnostic purposes in emergency surgery, though both resection and ovary sparing would need at least two trocars (one of them double channeled) to complete [14]. Single incision laparoscopic surgery and natural orifice transluminal endoscopic surgery are still controversial means of access as it seems the only advantage of those procedures is cosmesis. However, the main difficulty in single port surgery is suturing, which was not necessary in most cases of presented ovary procedures, which makes them open to this novelty [15]. Transgastric ovariectomy though feasible seems to be still in the experimental phase, being at least risky in children due to the smaller diameter of the child's esophagus [16]. The transvaginal approach is reserved for sexually active women, which makes it inappropriate in children.

Limited availability of gynecologists specializing in children and legal difficulties in access to operating gynecology wards for children make the coverage of ovarian pathology by a pediatric surgeon necessary. Unlimited cooperation with gynecologists in the diagnostic and post-operative care should be however assured.

False positive and false negative findings concerning ovarian pathology in the material are $33 \%$. Consequently, pediatric surgeons should be obliged to know how to deal with ovarian pathology and have legal authorization for such treatment $[17,18]$. It seems that it is easier to demand appropriate surgical treatment of ovarian pathology from a surgeon than an effective urgent procedure for appendicitis from a gynecologist.

The frequency of ovarian torsion of $2.5 \%$ in the general population operated on because of acute abdominal pain observed in the material seems to be too low to intervene surgically in every case of abdominal pain in girls, especially since $66 \%$ of lesions were detected on preoperative ultrasonography. The ovarian vascular supply is rich and comes from different sources, which results in organ survival time being longer than the seemingly analogous pathology that is torsion of the testicle [19]. However, with the increase in size of the cyst, the risk of ovary torsion and necrosis grows. So the au- thors are inclined to determine the size of the cyst above which there is an indication for surgery when symptoms arise. In this study, $5 \mathrm{~cm}$ in diameter was taken as the limit, which was consistent with the literature [20].

\section{Conclusions}

Summarizing the gained data described above, the laparoscopic treatment for ovarian masses is safe and efficient. The risk of a wrong preoperative diagnosis (ovary mass vs. appendicitis) is in any direction between $5 \%$ and $8 \%$, which is not high but still a significant number. The number large enough to be taken into consideration when surgical training and legislation is concerned.

It should be clearly stated also that the surgical treatment of lesions within the ovary in children should always be laparoscopic. The aim is to cut out the lesion, sparing ovarian parenchyma. One should always keep in mind some common symptoms of ovarian pathology and appendicitis and be prepared to change the procedure when forced by the intraoperative image.

\section{References}

1. Mayer JP, Bettolli M, Kolberg-Schwerdt A, et al. Laparoscopic approach to ovarian mass in children and adolescents: already a standard in therapy. J Laparoendosc Adv Surg Tech A 2009; 19 Suppl. 1: S111-5.

2. Spinelli C, Di Giacomo M, Cei M, Mucci N. Functional ovarian lesions in children and adolescents: when to remove them. Gynecol Endocrinol 2009; 25: 294-8.

3. Tessiatore P, Guanà R, Mussa A, et al. When to operate on ovarian cysts in children? J Pediatr Endocrinol Metab 2012; 25: 427-33.

4. Patel AG, Parker JE, Wallwork B, et al. Massive splenomegaly is associated with significant morbidity after laparoscopic splenectomy. Ann Surg 2003; 238: 235-40

5. Karpelowsky JS, Hei ER, Matthews K. Laparoscopic resection of benign ovarian tumours in children with gonadal preservation. Pediatr Surg Int 2009; 25: 251-4.

6. Shapiro EY, Kaye JD, Palmer LS. Laparoscopic ovarian cystectomy in children. Urology 2009; 73: 526-8.

7. Ates M, Sevil S, Bulbul M. Routine use of laparoscopy in patients with clinically doubtful diagnosis of appendicitis. J Laparoendosc Adv Surg Tech A 2008; 18: 189-93.

8. Abbas TO, Hayati A, Ali M. Role of laparoscopy in non-trauma emergency pediatric surgery: a 5-year, single center experience a retrospective descriptive study with literature review. BMC Res Notes 2012; 5: 550.

9. Murawski M, Gołębiewski A, Sroka M, Czauderna P. Laparoscopic management of giant ovarian cysts in adolescents. Videosurgery Miniinv 2012; 7: 111-3. 
10. Ateş O, Karakaya E, Hakgüder G, et al. Laparoscopic excision of a giant ovarian cyst after ultrasound-guided drainage. J Pediatr Surg 2006; 41: E9-11.

11. Wilczynski JR, Nowińska A, Szpakowski M, et al. Laparoscopic treatment of benign ovarian tumors. Ginekol Pol 2006; 77: 40-7.

12. Hayes-Jordan A. Surgical management of the incidentally identified ovarian mass. Semin Pediatr Surg 2005; 14: 106-10.

13. Spinelli C, Pucci V, Buti I, et al. The role of tumor markers in the surgical approach of ovarian masses in pediatric age: a 10-year study and a literature review. Ann Surg Oncol 2012; 19: 1766-73.

14. Gołębiewski A, Łosin M, Murawski M, et al. One, two or three port appendectomy - a rational approach. Videosurgery Miniinv 2013; 8: 226-31.

15. Bryks-Laszkowska A, Gołębiewski A, Czauderna P. Laparoscopic single port surgery nephrectomy in a child - initial experience. Videosurgery Miniinv 2012; 7: 304-6.

16. Ryska O, Martinek J, Filipkova T, et al. Single loop-and-clips technique (king closure) for gastrotomy closure after transgastric ovariectomy: a survival experiment. Videosurgery Miniinv 2012; 7: 233-9.

17. Partecke LI, von Bernstorff W, Karrasch A, et al. Unexpected findings on laparoscopy for suspected acute appendicitis: a pro for laparoscopic appendectomy as the standard procedure for acute appendicitis. Langenbecks Arch Surg 2010; 395: 1069-76.

18. Bowling CB, Lipscomb GH. Torsion of the appendix mimicking ovarian torsion. Obstet Gynecol 2006; 107: 466-7.

19. Celik A, Ergün O, Aldemir H, et al. Long-term results of conservative management of adnexal torsion in children. J Pediatr Surg 2005; 40: 704-8.

20. Oltmann SC, Fischer A, Barber R, et al. Cannot exclude torsion: a 15-year review. J Pediatr Surg 2009; 44: 1212-6.

Received: 28.08.2013, accepted: 24.11.2013. 